\title{
PEQUENO SURTO DE LEISHMANIOSE TEGUMENTAR AMERICANA EM MACUCO (CORDETRO - RJ)
}

\author{
Jacqueline A. Menezes, ** Vera Lúcia Lopes dos Reis *** e José Amsterdam Vasconcellos ***
}

\begin{abstract}
Por ocasiäo de um pequeno surto de leishmaniose tegumentar anericana em localidade do municipio de Cordeiro (RJ) foram documentados 14 casos. Entre os fatos que chamaram a atenção dos autores estão a alta ocorrência em crianças (57\%), a freqüência do componente linfangitico (40\%), a tendência à cicatrização espontânea e a distribuiçẫo peri-domiciliar dos casos.

Um inquérito pela intradermoreação de Montenegro na área de maior concentração de casos, realizado numa fazendo e numa escola, mostrou $17 \%$ de reações positivas, $17 \%$ de duvidosas e $66 \%$ de reações negativas, num total de 117 individuos testados. Não foram encontrados casos antigos cicatrizados, o que sugere um foco recente. Discute-se o significado das reaçóes duvidosas.
\end{abstract}

\section{INTRODUÇÃO}

A leishmaniose tegumentar é atualmente uma doença de ocorrência esporádica no Estado do Rio de Janeiro. Até 1970, os casos que tínhamos a oportunidade de observar no Serviço de Doenças Infecciosas e Parasitárias do Hospital Universitário Antonio Pedro, eram geralmente crônicos, com lesões mucosas e cicatrizes de lesões cutâneas antigas. Num trabalho anterior (4) realizamos um inquérito pela intradermoreação de Montenegro em pequena área do município de Trajano de Moraes, de onde provinham três dos nossos casos. Os resultados então obtidos confirmaram a nossa impressão inicial de que a leishmaniohe tegumentar era de ocorrência antiga no Estado e atualmente pouco freqüente.

Em agosto de 1971 o ambulatório de serviço de Dermatologia nos encaminhou dois pacientes, um pai e sua filha com lesões ulceradas de menos de 6 meses de evoluçāo, provenientes da localidade de $\mathrm{Ma}-$ cuco, no município de Cordeiro (Fig. 1), área geograficamente próxima à estudada no trabalho anterior. Foram encontrados mais 12 casos no local, todos de evolução inferior a um ano. Paralelamente, fizemos um pequeno inquérito pela intradermoreação de Montenegro no local onde se concentrava a maioria dos casos - uma fazenda e a escola da região. A descrição dos casos e os resultados do inquérito estão expostos no presente trabalho.

* Trabalho apresentado no IX Congresso da Sociedade Brasileira de Medicina Troplcal - Fortaleza, fevereiro de 1973.

** Auxillar de Ensino, Disc. de D. Infeccsiosas e Parasitárias Depto. de Medicina Clínica, U. F. Fluminense.

*** Residente do Serviço de Dermatologia e Sifiligrafia do Depto. de Clínica Medica da U.F.R.J.

*** Interno do Serviço de D. Int. Parasitárias to Depto. de M. Clínica da U. F. Fluminense Recebido para publicação em 28-4-74. 


\section{MATERIAL E MÉTODOS}

Os pacientes fgoram fichados, fotografados e biopsiados. Não foi feito o estudo parasitológico (isolamento de leishmanias em culturas ou por inoculação) devido a deficiências de ordem técnica na época. O diagnóstico foi baseado em evidências clínicas, histopatológicas e intradermoreação de Montenegro.

A maioria dos nossos casos - 5 em 14 - provinha de uma mesma fazenda (Fazenda do Sossêgo). O primeiro inquérito pela intradermoreação de Montenegro foi realizado nos habitantes desta fazenda, onde conseguimos testar 66 indivíduos. Numa segunda oportunidade, testamos 41 crianças na pequena escola onde estudam as que residem nesta área (ver esquema, Fig. 2). As 10 intradermoreações restantes foram :ealizadas em pacientes e familiares não incluídos nos inquéritos acima.

O antígeno usado para a intradermoreação foi o preparado pelo Instituto Oswaldo Cruz, cuja concentração é aproximadamente de 10 milhōes de parasitos (promastigotos) por $\mathrm{cm}^{3}$; a técnica foi a clássica com leitura 48 a 72 horas após. Eram considerados positivos os casos com induração igual ou superior a $5 \mathrm{~mm}$, duvidosos os casos com induração perceptível ao tato, porém inferior a $5 \mathrm{~mm}$ e negativos os casos sem induração. Não achamos útil discriminar o grau de positividade das reações (fraco, moderado ou forte) devido ao pequeno número global de positivos.

Ao realizarmos a leitura das reações indagávamos da residência e da naturalidade dos indivíduos, bem como da presença de lesōes dermatológicas ou cicatrizes. Consideramos como lesões suspeitas mínimas, lesões pápulo ou pústulo-crostosas semelhantes a um impetigo ou estrófulo banal que a experiência mostra poderem ser devidas a leishmaniose: não foram aqui estudadas pois regrediram espontâneamente a curto prazo. Foram registradas apenas para confronto posterior.

A relativa imprecisão das informações sobre o local de moradia das pessoas testadas - fato comum em área rural -, a sua grande mobilidade dentro de uma área relativamente pequena (cerca de $31 \mathrm{~km}^{2}$ no triângulo compreendido entre Macuco, a fazendo do Sossêgo e o Val de Palmas), ao lado da seleção prévia por nós efetuada ao realizarmos os testes na área de maior concentração dos casos, nāo nos permitiram tentar uma correlação geográfica entre os Montenegro-positivos e a procedência dos doentes.

\section{RESULTADOS}

\section{A - Estudo dos casos}

Foram examinados ao todo 14 pacientes (Quadro I); a distribuição por sexo' e faixa etária está resumida no Quadro II: dos pacientes adultos, 4 eram do sexo masculino e 2 do sexo feminino; havia 8 crianças entre as quais uma menina de 9 meses cuja lesão vimos evoluir em poucas semanas de uma pequena pápula crostosa, à qual a própria mãe não deu valor, a uma úlcera de uns $4 \mathrm{~cm}$ de diâmetro (Fig. 3) na região pré-auricular.

o caso mais antigo tinha cerca de um ano de 'evolução e o mais recente era o da criança de 9 meses já citada, cuja lesão na nossa primeira visita tinha apenas uma semana de evolução.

As lesões na sua maioria eram as clássicas úlceras com bordos elevados e fundo granuloso em número de uma a três localizadas em ordem de freqüência, nos membros, face e tronco (Quadro I). Em 6 casos havia nítido componente linfangítico com cordão e gânglios linfáticos visiveis e palpáveis na zona de drenagem de lesão.

Em 5 casos evidenciamos a presença de parasitos na lesão por exame direto e/ou biópsia. A histopatologia mostrou, nos 8 casos em que foi estudada, ulceração por vezes acompanhada de hiperceratose $e$ acantose e, na derme, infiltrado do tipo granulomatoso linfo-histioplasmocitário com células epitelióides e número variável de células gigantes, geralmente do tipo Langhans; os parasitos, quando presentes, estavam livres e dentro de histiócitos.

A intradermoreação de Montenegro foi positiva em 12 dos 14 casos. Num dos pacientes (com parasitos na biópsia) não foi realizada por motivos de ordem técnica e, no caso restante, ela foi duvidosa apesar da lesão, rica em parasitos, ter mais de 3 meses de evolução. Na maioria dos pacientes a reação se mostrou fortemente 


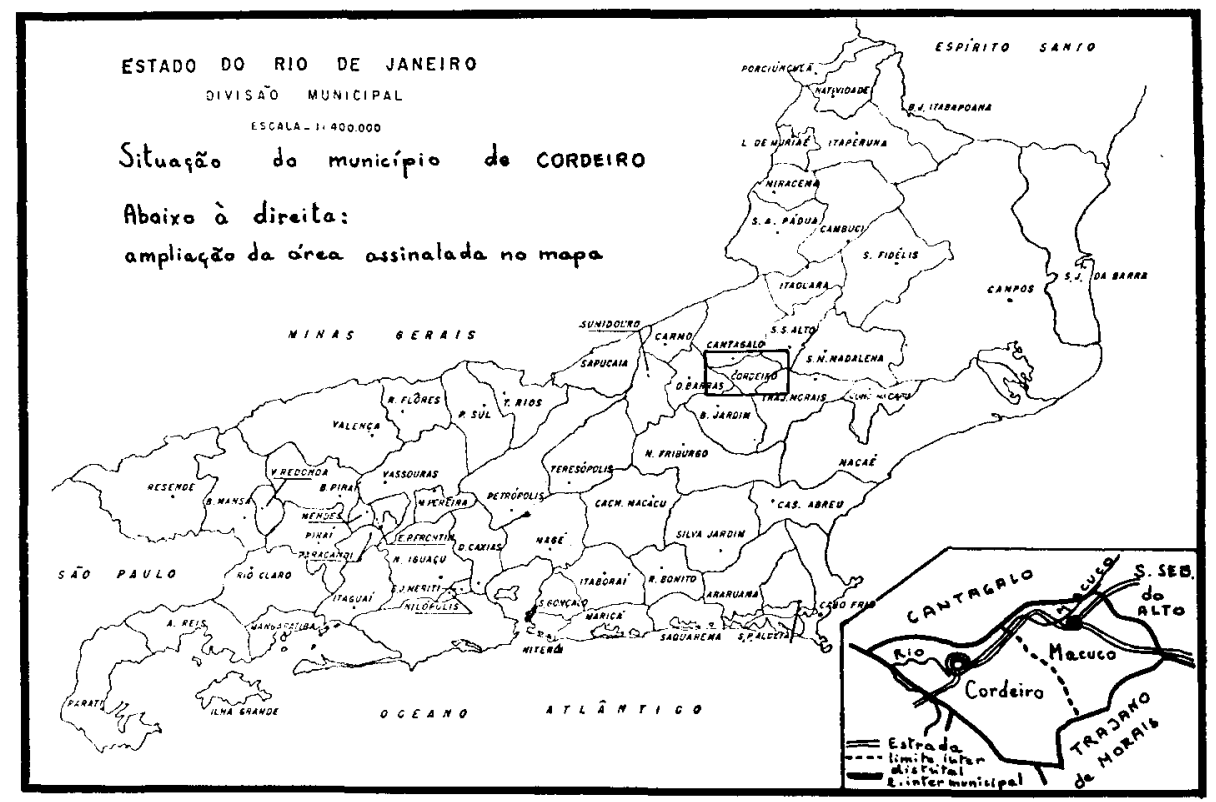

Fig. 1 - Situaçāo do município de Cordeiro, Estado do Rlo de Janelro.

EROQUI DA ÁREA DO OISTRITO

de macUCO-MUNICIPIO de tordeIRO-RJ

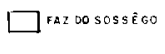

ÁREA DE SURTO DE Leishmonios.

NO DISTRITO DE MACUCO

MUNICIPIO DE CORDEIRO.

ESTADO DO RIO DE JANEIRO

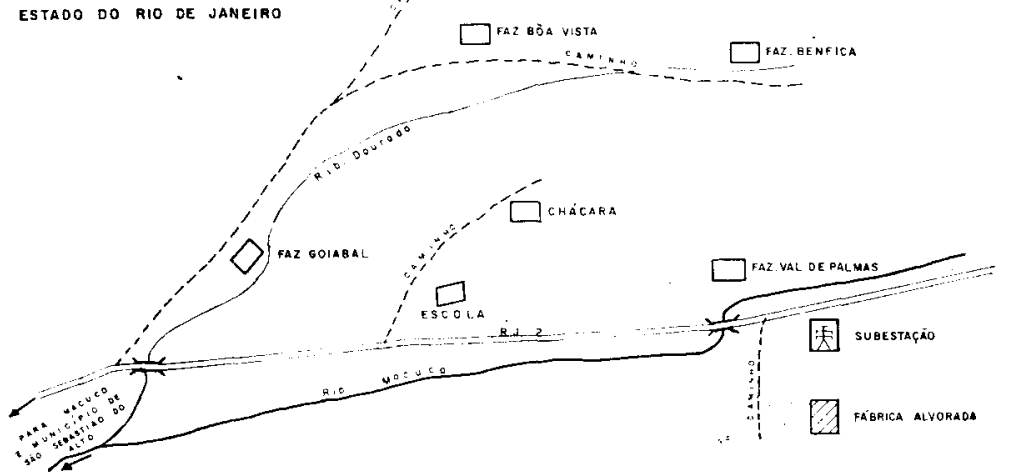

Fig. 2 - Area de surto de letshmaniose no distrito de Macuco, municipio de Cordetro. 


\section{QUADRO I}

\section{CASOS DE LEISHMANIOSE TECUMENTAR}

MACUCO - CORDEIRO - R-

1971

\begin{tabular}{|c|c|c|c|c|c|c|c|c|c|c|}
\hline \multirow{2}{*}{ Caso N.o } & \multirow{2}{*}{\multicolumn{4}{|c|}{ Identificação }} & \multirow{2}{*}{ Procedência } & \multirow{2}{*}{$\begin{array}{c}\text { Tempo } \\
\text { evolução }\end{array}$} & \multicolumn{4}{|c|}{ Lesões } \\
\hline & & & & & & & N.o & local. & $\operatorname{linf}$. & parasito \\
\hline 1 & o.s. & masc. & 40 & a & Sossego & 6 meses & 2 & membro sup. & $\mathbf{P}$ & $\mathrm{N}$ \\
\hline 2 & M.S. & fem. & 12 & & " & 6 meses & 1 & face & $\mathrm{P}$ & $\mathbf{N}$ \\
\hline 3 & E.S. & masc. & 10 & $\mathrm{a}$ & $"$ & 4 meses & 1 & orelha & $\mathbf{P}$ & $\mathbf{N}$ \\
\hline 4 & M.S. & fem. & 8 & $\mathrm{a}$ & $"$ & 1 ano & 1 & membro inf. & $\mathrm{N}$ & - \\
\hline 5 & M.RS. & fem. & 10 & a & " & 6 meses & 2 & membro sup. & $\mathbf{N}$ & - \\
\hline 6 & M.FS. & fem. & 24 & $\mathrm{a}$ & Boa Vista & 3 meses & 2 & membro sup.; face & $\mathbf{N}$ & $\mathbf{N}$ \\
\hline 7 & S.FS. & fem. & 9 & $\mathrm{~m}$ & $"$ & 1 semana & 1 & face & $\mathbf{P}$ & $\mathbf{P}$ \\
\hline 8 & MA.C. & fem. & 11 & $\mathrm{a}$ & Benfica & 3 meses & 3 & membro inf. & $\mathbf{P}$ & $\mathbf{P}$ \\
\hline 9 & LF.R. & fem. & 50 & a & Val PaImas & 3 meses & 2 & membro sup.; face & $\mathbf{P}$ & $\mathbf{P}$ \\
\hline 10 & P.C. & masc & 41 & $\mathrm{a}$ & $"$ & 3 meses & 1 & face & $\mathrm{N}$ & $\mathbf{p}$ \\
\hline 11 & PC.C. & masc. & 18 & a & - & 4 meses & 1 & membro inf. & $\mathrm{N}$ & - \\
\hline 12 & PS.M. & masc & 12 & a & Macuco & 7 meses & 3 & membro sup. tronco & $\mathrm{N}$ & - \\
\hline 13 & D.B. & fem. & 12 & a & S. Seb. Alto & 3 meses & 3 & membro inf. & $\mathrm{N}$ & $\mathbf{N}$ \\
\hline 14 & RJ.M. & masc. & 26 & a & & 6 meses & 1 & tronco & $\mathrm{N}$ & $\mathbf{P}$ \\
\hline
\end{tabular}

Abreviações: local.: localização

linf.: linfangite

$\mathrm{P}$ : positivo.

N: negativo. 


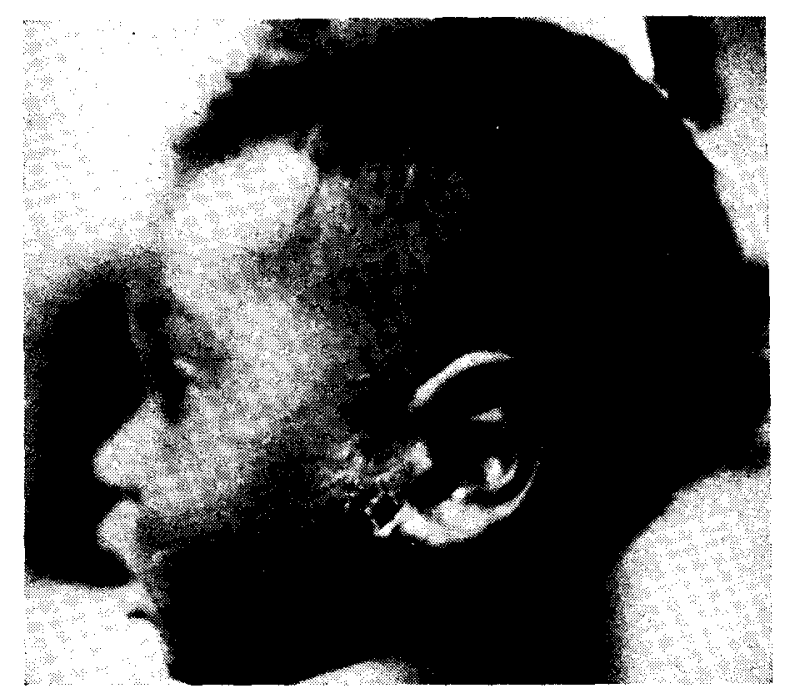

Fig. 3 - Uiceraçăo de cerca de $4 \mathrm{~cm}$ na regiāo pré-
auricular.

positiva, isto é, com área de induração igual ou superior a $12 \mathrm{~mm}$ e muitas vezes com a presença de vesícula ou necrose central.

Dez dos casos eram de áreas geograficamente muito próximas (Fig. 2): Fazenda do Sossêgo ( 5 casos) Boa Vista ( 2 casos), Val de Palmas (2 casos) e Benfica (1 caso). Um paciente residia no centro de Macuco, um tinha domicílio variável e dois provinham de área fronteiriça no município vizinho de são Sebastião do Alto. Quatro dos cinco casos da fazenda do Sossêgo pertenciam a uma única familia (pai e 3 crianças), residindo, portanto, na mesma casa. Os 2 casos de Boa Vista eram mãe e filha.

\section{$\mathrm{B}$ - Inquérito pela intraderhoreação de Montenegro}

Realizamos um total de 117 reações: 53 em indivíduos do sexo masculino e $64 \mathrm{em}$ indivíduos do sexo feminino: 66 eram adultos e os demais tinham idade inferior ou igual a 12 anos (Quadro III).

Obtivemos um total de 20 reações positivas $(17 \%)$ entre as quais estavam 12 dos nossos pacientes, 2 apresentavam lesōes suspeitas minimas não confirmadas e 6 não apresentavam lesão alguma. Não encontramos pessoas com Montenegro positivo que apresentassem cicatrizes sugestivas e/ou história compatível com lesão antiga.
O número de reações duvidosas foi também de $20(17 \%)$. Entre estas, uma era de um paciente nosso com lesão rica em leishmanias, 4 apresentavam lesôes mínimas do tipo impetigo e os demais não apresentavam lesão alguma. Aqui novamente não encontramos individuos portadores de cicatrizes e/ou história sugestiva. 77 reações foram negativas $(66 \%)$.

\section{DISCUSSÃO}

Fatos que chamaram a nossa atenção no presente trabalho:

1) O grupamento familiar (peri-domiciliar dos casos bem como a sua concentração numa área geograficamente pequena (estimada em cerca de $31 \mathrm{~km}^{2}$ ), a distribuição relativamente homogênea por sєxo e faixa etária, com c acometimento de crianças $(57 \%)$.

2) Aspectos clínicos: - a evolução rápida na criança peouena (c. 7); a tendência à cicatrização espontânea das lesões cutâneas a longo prazo (principalmente quando localizadas nos membros inferiores) como evidenciado em 3 dos nossos casos (c. 4, 5 e11) que não puderam ser tratados $\mathrm{n}$ época por escassez de medicamento; a freqüência do componente linfangítico, muito nítido em 6 dos nos- 


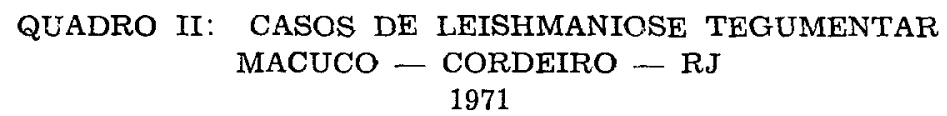

Distribuição por sexo e faixa etária

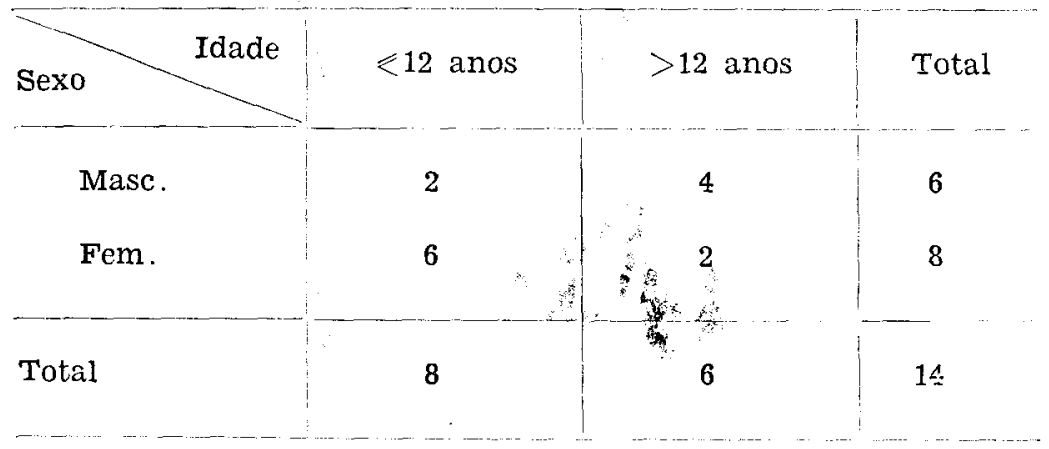

\section{QUADRO III: INQUÉRITO PELA INTRADERMOREAÇĀO DE MONTENEGRO}

Distribuição por sexo e faixa etária

\begin{tabular}{l|c|c|c}
\hline Sexo & Idade & $>12$ anos & Total \\
Masc. & 28 & 25 & 53 \\
Fem. & 36 & 26 & 64 \\
\hline Total & 66 & 51 & 117 \\
\hline
\end{tabular}

sos casos $(40 \%)$, que, salvo num caso, era difícil de atribuir à infecção secundária.

3) A não evidenciação de parasitos em 5 aos 10 casos em que tivemos a oportunidade de pesquisá-los. Isto poderia ser devido a deficiências técnicas na época, porém, com nossa experiência atual, temos a impressão de que a doença, no Estado do Rio, é de caráter pauci-parasitário com intensa reação tecidual.
4) Os casos tendem a não ser notificados: a pouca freqüência da doença no nosso Estado, aliada à tendência à cicatrização espontânea das lesões e à relativa dificuldade de diagnóstico laboratorial (pobreza em parasitos), fazem com que a leishmaniose tegumentar na sua fase cutânea seja raramente suspeitada e notificada no nosso meio. No surto em estudo, apenas dois casos haviam sido encaminhados a serviço médico especializado. O restante foi desco- 
berto no local através da indicacāo dos primeiros e da reação intradérmica. É preciso lembrar que o próprio paciente, numa mentalidade tipicamente rural, não valoriza a sua doença na medida em que as lesões não o impedem de trabalhar; quando, por acaso, procura um serviço de assistência, recebe, na maioria das vezes, apenas um tratamento local. A incidência da doença poderia ser, portanto, maior do que parece a primeira vista.

5) No Gráfico I agrupamos os resultados da intradermoreação de Montenegro, em correlação com a presença de lesões. Notamos logo que a maioria das reações positivas pertence a casos de lesões leishmanióticas ativas, ao passo que as reações duvidosas colocam-se aqui numa distribuição semelhante à das reações negativas. Por outro lado, não encontramos reaçōes positivas ou duvidosas em portadores de cicatrizes com histónia sugestiva de lesões antigas.

Estes resultados vão em sentido contrário ao que havíamos observado no inquérito realizado no ano anterior no município vizinho de Trajano de Moraes (4), onde não encontramos lesāo típica ativa entre os Montenegro-positivos mas onde notamos a ocorrência freqüente de cicatrizes e história sugestiva datando às vezes de 20 anos ou mais. Por outro lado, naquela área, havia uma certa correlação entre os Montenegros positivos e os duvidosos.

Ressaltamos aqui a diversidade de situaçōes: no caso de Trajano de Moraes, um município onde, em alguns pontos, a leishmaniose ocorria de modo esporádico há longo tempo e, no presente caso, a ocorrência epidêmica da doença em área previamente poupada, com população desprovida de imunidade. Tal tipo de situação foi descrita no Estado de São Paulo, por Pessoa (6) em 1941 onde era fácil de correlacionar com o tempo de colonização dos núcleos habitacionais e a recente ou não derrubada de matas, fato que não se verifica no nosso caso: grandes derrubadas recentes foram negadas por todas as pessoas interrogadas a respeito.

Significado das reações duvidosas - A maioria dos autores computa os resultados dos inquéritos por intradermoreação de Montenegro, dividindo-se basicamente em reações positivas, negativas e duvidosas. O critério para a reação duvidosa, chamada de fracamente positiva por alguns - Pessoa e Lopes (8), Pifano (9) -, consiste em eritema sem pápula ou pápula menor de $5 \mathrm{~mm}$ mais perceptivel ao tato, e é bastante universal - Aston e Thorley (1), Hómez e Romero (3), Nery-Guimarães (5). O seu significado é entretanto altamente discutido. Barbosa $\mathrm{e}$ cols. (2), utilizando antígeno de Leptomonas pessoai de potência comparável ao preparado com $L$. brasiliensis, discutem extensamente o significado destas reações que definem como reações específicas fracas, concluindo que têm valor apenas epidemiológico e podem ser atribuídas a infecções antigas. Hómez e Romero (3), num

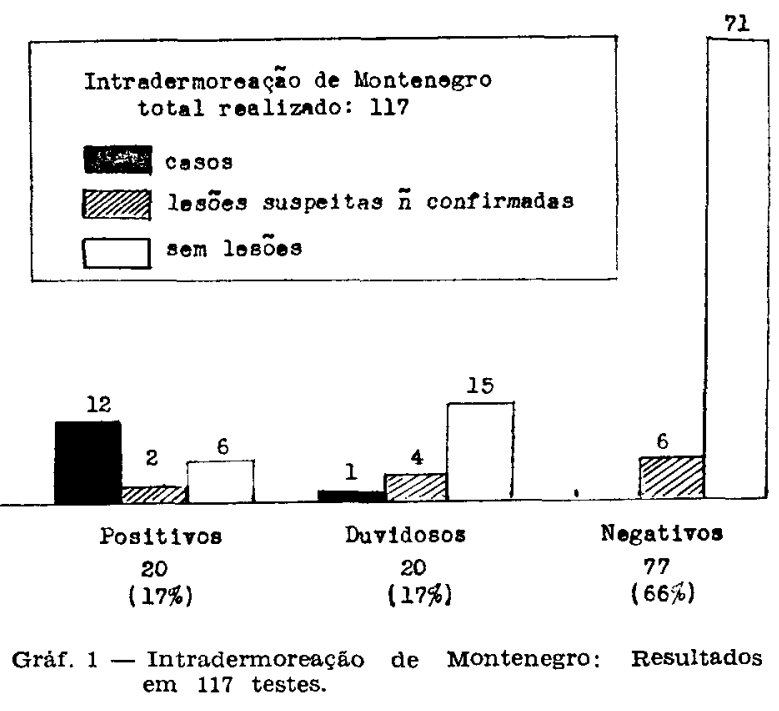


inquérito realizado em 3 localidades da Venezuela, notam a correlação maior das reações duvidosas com as negativas do que com as positivas, à semelhança do observado no presente trabalho. Aston e Thorley (1), testando índios de tribos do Parque Nacional do Xingu, desprezaram no cômputo dos resultados as reações duvidosas por considerarem-nas desprezíveis em número. Já Pessoa e Lopes (8) com o nome de "fra-. camente positivas" incluiram-nas entre as reações positivas no inquérito realizado em Jacobina (BA) em 1962.

Achamos extremamente dificil qualquer comparação entre estes trabalhos, por não ser $o$ antígeno de Montenegro padronizado e variar enormemente em termos de método de preparo e aferição e em concentração. Não é nossa intenção discutir este item mais detalhadamente no âmbito deste trabalho; vamos apenas lembrar algumas discrepâncias. O antígeno que usamos, preparado pelo Instituto Oswaldo Cruz, contém aproximadamente $10 \mathrm{mi}-$ lhões de formas promastigotas por $\mathrm{cm}^{3}$. Esta é também a concentração recomendada por Rotberg (10) que estudou detalhadamente o assunto. Pessoa no seu compêndio de Parasitologia Médica (7) cita a concentração de 2 a 3 milhões de leptomonas por $\mathrm{cm}^{3}$, mas o antígeno usado por Pessoa e Lopes no trabalho já citado contém 5 milhões de leptomonas for $\mathrm{cm}^{3}$. O antígeno usado por Aston e Thorley é o preparado pelo Instituto Evandro Chagas de Belém, que sabemos conter aproximadamente 2 milhōes de formas promastigotas por $\mathrm{cm}^{3}$. Quanto ao antigeno venezuelano, é aferido por nefelometria (escala 2 de Mac Farlane) e "titulado clinicamente" em indivíduos de sensibilidade conhecida segundo Pifano (9), o antígeno utilizado por Barbosa e cols., preparado no Instituto de Patologia Tropical da U.F.GO a partir de Leptomonas pessoai é aferido por nefelometria de maneira a alcançar a escala 5 de Mac Farlane.

Cada centro universitáário e de pesquisa prepara hoje seu próprio antígeno com muitas melhorias no sentido de purificação e modo de aferição; seria, porém, desejável que fossem, de alguma maneira, padronizáveis, - utilização de cepa padrão, modo de preparo, aferição e titulagem idênticos - para que se pudessem algum dia reunir e comparar os resultados obtidos por varios pesquisadores em pontos diversos do País.

\section{SUMMARY}

During a small outbreak of American mucocutaneous leishmaniasis in a village of the municipality of Cordeiro, state of Rio de Janeiro, fourteen cases were documented. Outstanding features were the high incidence in children (57\%), the clinical evidence of lymphangitis in $40 \%$, the tendency towards spontaneous healing in the less recent cases and the peri-domestic distribution of the cases. A Mcntenegro skin-test survey carried out in 117 persons yielding $17 \%$ positive and $17 \%$ doubtful reactions. No positive reactors with old scars or signs of mucosal envolvement were noted, this being probably a recent focus. The significance of doubtjul skin reactions is discussed.

\section{REFERENCIAS BIBLIOGRÁFICAS}

1. ASTON, D.L. \& THORLEY, A.P. Leishmaniasis in Central Brazil: results of a Montenegro skin test survey among Amerindians in the Xingú National Park. Trans. R. Soc. trop. Med. Hyg. 64: 671-678, 1970.

2. BARBOSA, W., SOUSA, M.C.M., RASSI, D.M., OLIVEIRA R.L. \& MOTA, L. - Investigação sobre imuno- logia da leishmaniose tegumentar americana - I. Intradermoreação de Montenegro concomitante com antígenos de Leptomonas pessoai e brasiliensis. Rev. Pat. Trop. 1: 377-383, 1972.

3. HÓMEZ, J. \& ROMERO, H.M. - Investigación inmunoalergica con leishmanina em tres localidades del Esta- 
do Zulia (Venezuela). Kasmera 3: 281-297, 1970.

4. MENEZES, J.A., REIS, V.L.L. \& COURA, J.R. - Inquérito preliminar pela intradermoreação de Montenegro em população rural do município de Trajano de Moraes - RJ. Rev. Soc. Bras. Med. Trop. VI: 171-176, 1972.

5. NERY-GUIMARÃES. F. - Estudo de um foco de leishmaniose muco-cutânea na Baixada Fluminense (Estado do Rio de Janeiro). Mem. Inst. Osw. Cruz 53: 1-11, 1955.

6. PESSOA, S.B. - Indices de disseminação da leishmaniose tegumentar em algumas zonas novas do Estado de Sẫo Paulo. Rev. Biol. Hyg. 11: 1-9, 1941.
7. PESSOA, S.B. - Parasitologia Médica - Editora Guanabara Koogan, Rio 1969 .

8. PESSOA, S.B. \& SOUZA LOPES, J.A. - Sobre a intradermoreação de Montenegro em região endêmica de leishmaniose tegumentar e visceral. Rev. Inst. Med. Trop. S. Paulo 5: 170$-175,1963$.

9. PIFANO, F - La evaluación de la leishmaniasis tegumentaria americana en el Valle de Aroa, Estado Yaracuy, mediante el indice alergico. Arch. Venez. Med. Trop. Parasit. Med. IV: 25-35, 1962 .

10. ROTBERG, A. - Contribuição para o estudo da alergia na leishmaniose tegumentar americana. Rev. Hosp. N. S. Aparecida 5: 3-83, 1952. 